\section{Effect of the COVID-19 Pandemic on Neurology Trainees' Education and Practice Amtul Farheen, $\mathrm{MD}^{1}$; James Grogan, $\mathrm{MD}^{1}$; William K. Jens, DO; Christina Maxwell, PhD, MTR²; Karima Benameur, $\mathrm{MD}^{3}$ \\ ${ }^{1}$ Department of Neurology, Penn State Hershey Medical Center \\ ${ }^{2}$ Global Neurosciences Institute, Pennington, NJ \\ ${ }^{3}$ Department of Neurology, Emory University}

Keywords: PPE, COVID-19, resident, trainee, education

\section{ABSTRACT}

Glossary: PPE = Personal Protective Equipment; COVID-19 = Coronavirus Disease 2019.

\section{Introduction}

Since December 2019, the Severe Acute Respiratory Syndrome Coronavirus 2 (SARS-CoV-2), that spreads by close proximity between humans ${ }^{1}$, has produced a global pandemic known as COronaVIrus Disease-19 (COVID-19) ${ }^{2}$. The World Health Organization declared COVID-19 a global pandemic on March 11, 20203. In the United States, cases of COVID-19 have surpassed 6 million with more than 200,000 deaths ${ }^{4}$. The virus has had a significant impact not only on health care systems but nearly all aspects of life ${ }^{5}$. Residents and fellows are at the frontline of patient care at most academic and teaching hospitals with an increased risk of exposure to communicable diseases ${ }^{6}$. They face challenges with personal safety and well-being, disruptions in training and practice, and relationship strain?

Neurology resident education and service continued to be affected by the COVID-19 pandemic ${ }^{8}$. Trainees on inpatient rotations have been at the forefront of caring for afflicted patients, whereas the challenge in outpatient rotations has been the rapid adoption of telemedicine practices and transitioning to virtual visits9. The required social distancing has been affecting education, including daily resident lectures, grand rounds and conferences. Physical and emotional strain on trainees in New York City, an epicenter of the pandemic in the US early on, was palpable ${ }^{6}$.

As the pandemic unfolded, neurology trainees anecdotally reported modifications to their schedules, educational and academic activities, and administration of inpatient and outpatient services. This survey aimed at gathering data on the varied experiences by neurology trainees across the country.

\section{Methods}

The authors developed a self-administered, Englishlanguage survey of 36 questions using Google forms. The questions were developed based on consensus amidst the authors and focused on issues raised during the COVID-19 pandemic that warranted further exploration. The survey was sent to US-based trainees in neurology (residents and fellows) through a number of mechanisms including social media platforms (Facebook $\mathbb{C}$, Twitter $($ ) ), email list services (program director mailing lists, residency lists, and local state neurology societies), and the American Academy of Neurology (AAN) Synapse platform. Participation in the survey was voluntary and anonymous, and consent was obtained by agreeing to participation. The target audience was any active resident or fellow in neurology or a neurology subspecialty. The survey was open online from June 27, 2020 to August 18, 2020.

Questions included basic demographics (age, sex, state, graduate year training), practice and education before the pandemic, and practice changes due to the pandemic (telemedicine, use of personal protective equipment (PPE), institutional guidelines, impact of the pandemic on the wellbeing of trainees).

Potential confounders included the possibility that the survey could be taken twice by the same individualgiven desire to preserve anonymity, we chose not to track respondents or require sign-in but encouraged respondents to only take the survey once. Missing data points were handled by reducing the total number of respondents as applicable. However, there were very few relevant missing data points. One respondent did not check "yes" in the consent-landing page and that response was not included in the final dataset.

\section{Data Availability}

The complete survey and the raw anonymized data will be shared by request from any qualified investigator.

\section{Standard Protocol Approvals}

The study protocol was reviewed by the Emory Institutional Review Board and was deemed to meet criteria for exemption under 45 CFR 46.104(d)(2). The study was approved for indefinite exemption, barring significant changes in the protocol. 
Table 1. Demographics $(\mathrm{N}=285)$

\begin{tabular}{|l|l|l|l|l|l|}
\hline Sex $^{\mathrm{a}}$ & Male & & & & Female \\
\hline Training Status & $91(32 \%)$ & & & & $192(67 \%)$ \\
\hline & PGY2 & PGY3 & PGY4 & Fellow & Child Neurology \\
\hline Setting & $57(20 \%)$ & $75(26.3 \%)$ & $76(26.6 \%)$ & $50(17.5 \%)$ & $27(9.5 \%)$ \\
\hline & Academic & & & & Community \\
\hline
\end{tabular}

${ }^{a}$ One respondent preferred not to answer, one non-binary

\section{Results}

286 US trainees participated in the survey. One participant did not consent and was excluded. Of the 285 trainees whose responses were analyzed, two-thirds (67\%) identified as female, and almost all (93\%) practiced in a university setting. Thirty-four states and the District of Columbia were represented with the most respondents from California (35/285, 12\%), Pennsylvania (31/285, 11\%), Georgia (29/285, 10\%), and New York (23/285, 8\%). There was an even breakdown of responses by post-graduate year as shown Table 1.

\section{Telemedicine}

More than half of trainees (60\%) reported telemedicine being available prior to the pandemic at their institutions, used mainly in the inpatient setting for stroke care (45\%). Among trainees, only $13 \%$ reported using these available telemedicine platforms prior to the pandemic. Up to $84 \%$ did not have training in telemedicine prior to the pandemic.

After the onset of the pandemic, the vast majority (91\%) of respondents reported using telemedicine as a medium for patient care. Telemedicine was used in the outpatient setting (48.1\%) and combined outpatient and inpatient (43.2\%) settings. In the outpatient setting, 70\% of respondents reported using telemedicine for general neurology visits and 54\% reported using it for subspecialty neurology clinics.

The majority of trainees (78\%) reported using telemedicine for both new patients and follow up visits. Notably, only $42 \%$ received education in telemedicine including how to perform a neurological examination. Despite this, 50\% (143) trainees reported performing tele-neurological examinations including validated scales such as the NIHSS, UPDRS, MoCA as shown in figure 1. Supervision by an attending was synchronous during the tele-visit in most instances (57.6\%), however $41.5 \%$ reported asynchronous supervision, and less than $1 \%$ reported no supervision at all.

\section{Impact on Practice}

Respondents were surveyed regarding alterations to inpatient and outpatient schedules. Almost a third of trainees (33.6\%) reported a decrease in inpatient schedules, while more than half reported no change (29\%) or increase (28\%) in inpatient schedules. More than half (56\%) reported a reduced clinic schedule in the outpatient settings, about $11 \%$ reported increase in clinic schedule while about one third (32\%) had no change in outpatient schedules.

These changes caused $32 \%$ of respondents to feel worried about losing their neurological examination skills, and $73 \%$ of respondents to feel that their patients' access to care was reduced by the pandemic.

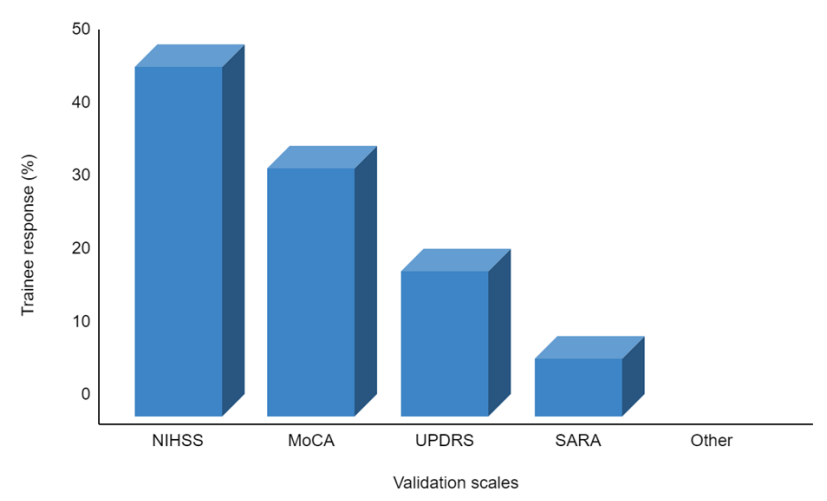

Figure 1. Validated scales used during telemedicine visits.

Breakdown of the validation scales used by $50 \%$ (143) trainees during televisits.

NIHSS = National Institutes of Health Stroke Scale, MoCA = Montreal Cognitive Assessment (MoCA), UPDRS = Unified Parkinson's Disease Rating Scale, SARA = Scale for Assessment and Rating of Ataxia, Other measures $(<1 \%)=$ MMSE, ABCD2, SLUMS, etc. 


\section{Impact on Education and Research}

A majority (77\%) of respondents believed that the pandemic affected their education. Almost half (48\%) noted fewer lectures, and virtually all respondents (98\%) reported transition of lectures to telephone or video-conferencing platforms.

Forty percent reported a reduction in grand rounds, and $17 \%$ reported that there were no grand rounds at all during the pandemic. Almost half (48\%) stated that morning rounds continued at a similar frequency but in a virtual setting, while 33\% indicated less frequent morning rounds. $77 \%$ of trainees accessed lectures outside their institutions, and $84 \%$ believed that tele-lecturing was an effective educational tool.

Among trainees involved in research, 48\% stated that their research continued during the pandemic uninterrupted, while $32 \%$ reported that their research activities were reduced.

\section{Impact on Trainees' Mental Health}

A majority $(75 \%)$ of respondents indicated that they experienced moderate to very high levels of additional stress during the pandemic. A positive trend was found between respondents reporting very high stress and increased inpatient workload (Pearson Chi Square 2l; $\mathrm{df}=4$; $\mathrm{p}<0.001$ ) (Figure 2).

When asked about PPE availability and institutional guidance about the pandemic, a third (33\%) reported that they had insufficient PPE (Table 2). Several respondents provided additional comments noting that during the onset of the outbreak, PPE was insufficient causing re-use of masks for weeks at a time, but as the pandemic went on, they were able to secure sufficient PPE.

A majority (64\%) of trainees felt somewhat to very unsafe, and almost all (95\%) of them reported feeling somewhat to greatly worried about exposing themselves and their families to COVID-19.

On a personal level, 38\% of trainee's stated needing childcare, and of these $33 \%$ described difficulty obtaining childcare during the pandemic.

\section{Discussion}

This is the first study to our knowledge looking at the impact of COVID-19 on neurology trainees across the United States. The responses of the trainees closely reflect much of the global experience with COVID-19, with rapid modifications to include quick implementation of telemedicine, remote online lectures, and personal feelings of uncertainty.

While in previous natural disasters training programs impacted by calamity were able to rely on the support of other institutions, a global pandemic with no unaffected region to support or relieve the pressures, has caused significant strain on the training system. Prior natural disasters like the Hurricane Katrina in 2005 in New Orleans disrupted medical training causing a drop in USMLE step $2 \mathrm{CK}$ scores ${ }^{10}$. In Texas, hurricane Harvey put trainees and their patients through significant stress with increased signs of burnout ${ }^{11}$.

As with previous disasters, resident practice patterns have been affected ${ }^{10,11}$. A majority of trainees have noted an overall decrease in clinical time. This reduction in clinical exposure is likely due to reduced overall clinical operations. This was partially mitigated by the implementation of telemedicine with a striking seven-fold increase in use of telemedicine among trainees. The rapidity of the pandemic engulfing the country however did not allow for enough time for proper training in telemedicine, with the majority of trainees (63\%) having to use this new technology without training, and in half the visits without proper synchronous

Table 2. Responses regarding stress and PPE availability

\begin{tabular}{|l|l|l|l|}
\hline Stress at Work & Minimal & Modest & Very High \\
\hline & $26 \%$ & $47 \%$ & $27 \%$ \\
\hline PPE availability & Yes & No & Other \\
\hline & $58 \%$ & $32 \%$ & $\begin{array}{l}\text { Variable at different points in } \\
\text { pandemic with difficulty in the initial } \\
\text { phase }\end{array}$ \\
\hline Feeling safe at work & To a great extent & Somewhat & Not at all \\
\hline & $36 \%$ & $58 \%$ & $6 \%$ \\
\hline Worry about exposing family & To a great extent & Somewhat & Not at all \\
\hline & $37 \%$ & $58 \%$ & $5 \%$ \\
\hline
\end{tabular}

PPE $=$ Personal Protective Equipment 
supervision by an attending. The AAN Telemedicine Work Group recommends comprehensive training in clinical bedside neurology for the safe practice of teleneurology ${ }^{12}$.

Neurology has had a longstanding history of using telemedicine, with the first telestroke pilot programs developed about twenty years ago $^{13,14}$, then expansion throughout multiple neurologic subspecialties ${ }^{15,16,17}$. Moreover the remote utilization of functional and outcome metrics has been validated, including the NIHSS ${ }^{18}$, stroke dysphagia screen ${ }^{19}$, modified $\mathrm{UPDRS}^{20}$, and $\mathrm{MoCA}^{21}$. Despite lack of training, most trainees were able to incorporate telemedicine into their patient care and remotely manage patients, ensuring continuity of care as much as possible. Development of more structured and focused training on telemedicine education is likely needed to better serve residents in the future.

In addition to the impact on bedside teaching, curricula and their method of delivery have been drastically affected as well. COVID-19 and associated social distancing practices have limited group lectures, cancelled or delayed major and minor conferences, and pushed many into the world of online learning. Our survey confirms that trainees report fewer lectures in addition to a reduced number of grand rounds and morning rounds lectures. Many trainees engaged in outside lectures, this could be related to increased availability of virtual grand rounds and other online accessible resources. While $84 \%$ found tele-lecturing an effective medium, this still represents a marked change in education style. Combining the reduced didactic education with reduced clinical exposure, the practical impact on training could be substantial.

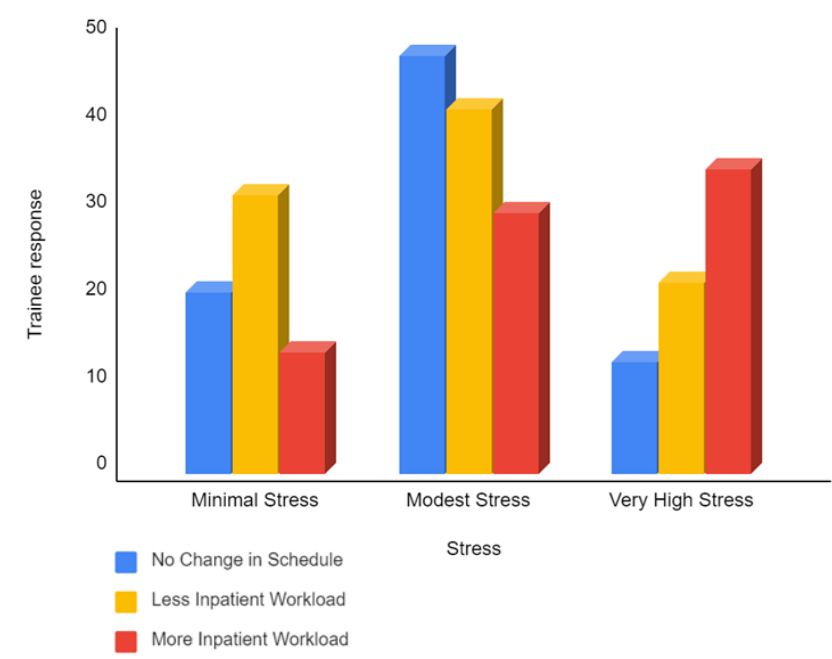

Figure 2. Correlation of stress levels among trainees with inpatient service.
As seen in previous natural disasters, we also note that residents suffered emotional distress. While there is no tangible environmental destruction as seen in previous disasters directly affecting housing, childcare, and physical structures, COVID-19 has caused significant economic and social disruption, as well as the constant looming threat of exposure to infection. The majority (75\%) of residents reported at least a moderate increase in stress. Increase in inpatient service load was a significant factor in increasing stress as seen in figure 2. Inadequate PPE was also felt to be a factor in increased stress and feeling unsafe. On a personal level, trainees noted challenges with childcare, concern of spreading COVID-19 to family at home, and simply increased overall stress.

\section{Limitations}

Our survey questions were developed with consensus amidst authors, which could be a limitation. Although we had a good representation of trainees, the number of survey participants was small, around $8 \%$ of neurology trainees ${ }^{22}$ (3643 US based combined residents, child neurology residents and subspecialty fellows per AAMC reports 20182019). Low response rate is possibly due to time requirement on behalf of trainees and survey availability on limited platforms. Female neurology trainees were overrepresented in our sample as the survey was posted on the Women's Neurologist Group on social media. We had representation from 34 states, even though California and New York had good response rates, together they represented $20 \%$ of the study sample. Another aspect is the timing of the survey as the stress level, use of technology was different in the initial phase of pandemic. As neurology training programs adjust policies and embrace technology and tele-neurology education, responses may change. We plan to send a repeat survey at a later time point to gather data on the changing practices and experiences of the neurology trainees.

\section{Conclusion}

This study demonstrates that neurology trainee clinical practice and education was significantly affected by the COVID-19 pandemic. Most trainees had no training in teleneurology prior to the pandemic, yet the majority of them were thrust into the world of telemedicine, often without much training. An overall negative impact was noted on trainee education and wellbeing, with reduced educational activities and increased stress. As the long-term effects of the pandemic on society, patient care, and medical training 
remain unknown, neurology training programs must remain adaptable and innovative to meet these changing needs.

Experience gained in this pandemic should lead to formulation of residency and fellowship curricula with optimal preparedness and safe practice measures for trainees. Programs should incorporate training on teleneurology examination techniques, including the use of validated scales such as the MoCA and modified UPDRS remotely. It is critical to ensure safety of trainees, provide adequate PPE, and ensure adequate mental health assessment and support for their wellness as mandated by ACGME. Incorporation of innovative and dynamic modes of education using technology, simulation labs, and video conferencing will promote safe education and preparedness in the event of future global catastrophes.

\section{Corresponding author:}

Amtul Farheen, MD

Email: farheenbright@gmail.com

Address: 1700 S. Lincoln Ave, Lebanon, PA, 17042

Phone: 717-272-6621; Ext 4294

\section{References}

1. Machhi J, Herskovitz J, Senan AM, et al. The natural history, pathobiology, and clinical manifestations of SARS-CoV-2 infections. J Neuroimmune Pharmacol 2020;15:359-386.

2. Estrada E. COVID-19 and SARS-CoV-2. modeling the present, looking at the future. Phys Rep 2020;869:1-51.

3. Sohrabi C, Alsafi Z, O'Neill N, et al. World health organization declares global emergency: A review of the 2019 novel coronavirus (COVID-19). Int J Surg 2020;76:71-76.e 2019 novel coronavirus (COVID-19). Int J Surg 2020;76:71-76.

4. https://covid.cdc.gov/covid-data-tracker/\# cases_ $_{-}$ casesinlast7days

5. Nicola M, Alsafi Z, Sohrabi C, et al. The socioeconomic implications of the coronavirus pandemic (COVID-19): A review. Int J Surg 2020;78:185-193.

6. Agarwal S, Sabadia S, Abou-Fayssal N, Kurzweil A, Balcer LJ, Galetta SL. Training in neurology: Flexibility and adaptability of a neurology training program at the epicenter of COVID-19. Neurology 2020;94:e2608-e2614.

7. Kwon YS, Tabakin AL, Patel HV, Backstrand JR, Jang TL, Kim IY, Singer EA. Adapting urology residency training in the COVID-19 era. Urology 2020;141:15-19.
8. AlGaeed M, Grewal M, Richardson PK, Leon Guerrero CR. COVID-19: Neurology residents' perspective. J Clin Neurosci 2020;78:452-453.

9. Grossman SN, Han SC, Balcer LJ, Kurzweil A, Weinberg H, Galetta SL, Busis NA. Rapid implementation of virtual neurology in response to the COVID-19 pandemic. Neurology 2020;94:1077-1087.

10. Crawford BE, Kahn MJ, Gibson JW, Daniel AJ, Krane NK. Impact of hurricane katrina on medical student academic performance: The tulane experience. Am J Med Sci 2008;336:142-146.

11. Yeo CJJ, Román GC, Kusnerik D, et al. Trainee responses to hurricane harvey: Correlating volunteerism with burnout. Front Public Health 2018;6:224.

12. Hatcher-Martin JM, Adams JL, Anderson ER, et al. Telemedicine in neurology:Telemedicine work group of the american academy of neurology update. Neurology 2020;94:30-38.

13. Levine SR, Gorman M. “Telestroke”: The application of telemedicine for stroke. Stroke 1999;30:464-469.

14. LaMonte MP, Bahouth MN, Hu P, etal. Telemedicine for acute stroke: Triumphs and pitfalls. Stroke 2003;34:725728.

15. Hanson RE, Truesdell M, Stebbins GT, Weathers AL, Goetz CG. Telemedicine vs office visits in a movement disorders clinic: Comparative satisfaction of physicians and patients. Mov Disord Clin Pract 2018;6:65-69.

16. Rasmusson KA, Hartshorn JC. A comparison of epilepsy patients in a traditional ambulatory clinic and a telemedicine clinic. Epilepsia 2005;46:767-770.

17. Geronimo A, Wright C, Morris A, Walsh S, Snyder B, Simmons Z. Incorporation of telehealth into a multidisciplinary ALS clinic: Feasibility and acceptability. Amyotroph Lateral Scler Frontotemporal Degener 2017;18:555-561.

18. Shafqat S, Kvedar JC, Guanci MM, Chang Y, Schwamm LH. Role for telemedicine in acute stroke. feasibility and reliability of remote administration of the NIH stroke scale. Stroke 1999;30:2141-2145.

19. Morrell K, Hyers M, Stuchiner T, et al. Telehealth stroke dysphagia evaluation is safe and effective. Cerebrovasc Dis 2017;44:225-231.

20. Abdolahi A, Scoglio N, Killoran A, Dorsey ER, Biglan KM. Potential reliability and validity of a modified version of the unified parkinson's disease rating scale that could be administered remotely. Parkinsonism Relat Disord 2013;19:218-221. 
21. Abdolahi A, Bull MT, Darwin KC, Venkataraman V, Grana MJ, Dorsey ER, Biglan KM. A feasibility study of conducting the montreal cognitive assessment remotely in individuals with movement disorders. Health Informatics J 2016;22:304-311.

22. https://www.aamc.org/data-reports/studentsresidents/interactive-data/report-residents/2019/tableb3-number-active-residents-type-medical-school-gmespecialty-and-sex

Appendix: Authors

\begin{tabular}{|l|l|l|}
\hline Name & Location & Contribution \\
\hline Amtul Farheen, M.D. & $\begin{array}{l}\text { Penn State Hershey Medical Center, } \\
\text { Hershey, PA }\end{array}$ & $\begin{array}{l}\text { Design and conceptualized study; survey } \\
\text { development; contributed to data analysis; } \\
\text { contributed to manuscript writing, revision } \\
\text { and created final draft }\end{array}$ \\
\hline James Grogan, M.D. & $\begin{array}{l}\text { Penn State Hershey Medical Center, } \\
\text { Hershey, PA }\end{array}$ & $\begin{array}{l}\text { Survey development; contributed to } \\
\text { manuscript writing, analysis and revision }\end{array}$ \\
\hline William K. Jens, D.O. & $\begin{array}{l}\text { Penn State Hershey Medical Center, } \\
\text { Hershey, PA }\end{array}$ & $\begin{array}{l}\text { Survey development; contributed to } \\
\text { manuscript writing, analysis and revision }\end{array}$ \\
\hline Christina Maxwell, PhD & $\begin{array}{l}\text { Global Neurosciences Institute, Pennington, } \\
\text { NJ }\end{array}$ & $\begin{array}{l}\text { Survey development; performed majority } \\
\text { of data analysis and interpretation and } \\
\text { contributed to manuscript }\end{array}$ \\
\hline Karima Benameur, M.D. & Emory University, Atlanta, GA & $\begin{array}{l}\text { IRB process; survey design and development; } \\
\text { contributed to manuscript writing, data } \\
\text { analysis and manuscript revision }\end{array}$ \\
\hline
\end{tabular}

